\title{
TMT-based proteomic and bioinformatic analyses of human granulosa cells from obese and normal-weight female subjects
}

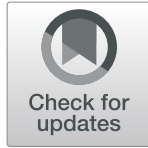

\author{
Chenchen $\mathrm{Si}^{1,2+}$, Nan Wang ${ }^{1 \dagger}$, Mingjie Wang ${ }^{2}$, Yue Liu' ${ }^{1}$ Z Zhihong $\mathrm{Niu}^{2 *}$ and Zhide Ding ${ }^{1 *}$
}

\begin{abstract}
Background: Increasing evidence supports a relationship between obesity and either infertility or subfertility in women. Most previous omics studies were focused on determining if the serum and follicular fluid expression profiles of subjects afflicted with both obesity-related infertility and polycystic ovary syndrome (PCOS) are different than those in normal healthy controls. As granulosa cells (GCs) are essential for oocyte development and fertility, we determined here if the protein expression profiles in the GCs from obese subjects are different than those in their normal-weight counterpart.

Methods: GC samples were collected from obese female subjects $(n=14)$ and normal-weight female subjects $(n=$ 12) who were infertile and underwent in vitro fertilization (IVF) treatment due to tubal pathology. A quantitative approach including tandem mass tag labeling and liquid chromatography tandem mass spectrometry (TMT) was employed to identify differentially expressed proteins. Gene Ontology (GO) and the Kyoto Encyclopedia of Genes and Genomes (KEGG) analyses were then conducted to interrogate the functions and pathways of identified proteins. Clinical, hormonal, and biochemical parameters were also analyzed in both groups.
\end{abstract}

Results: A total of 228 differentially expressed proteins were noted, including 138 that were upregulated whereas 90 others were downregulated. Significant pathways and GO terms associated with protein expression changes were also identified, especially within the mitochondrial electron transport chain. The levels of free fatty acids in both the serum and follicular fluid of obese subjects were significantly higher than those in matched normalweight subjects.

Conclusions: In GCs obtained from obese subjects, their mitochondria were damaged and the endoplasmic reticulum stress response was accompanied by dysregulated hormonal synthesis whereas none of these changes occurred in normal-weight subjects. These alterations may be related to the high FFA and TG levels detected in human follicular fluid.

Keywords: Granulosa cells, Proteomic analysis, Obesity, Free fatty acids, Electron transport chain, Mitochondria

\footnotetext{
* Correspondence: kangniu@sina.com; zding@shsmu.edu.cn

${ }^{+}$Chenchen Si and Nan Wang contributed equally to this work.

${ }^{2}$ Department of Gynecology and Obstetrics, Reproductive Medical Center,

School of Medicine, Ruijin Hospital, Shanghai Jiao Tong University, 197 Ruijin

2nd Road, 200025 Shanghai, China

'Department of Histology, Embryology, Genetics and Developmental Biology,

Shanghai Key Laboratory for Reproductive Medicine, School of Medicine,

Shanghai Jiao Tong University, 200025 Shanghai, China
}

(C) The Author(s). 2021 Open Access This article is licensed under a Creative Commons Attribution 4.0 International License, which permits use, sharing, adaptation, distribution and reproduction in any medium or format, as long as you give appropriate credit to the original author(s) and the source, provide a link to the Creative Commons licence, and indicate if changes were made. The images or other third party material in this article are included in the article's Creative Commons licence, unless indicated otherwise in a credit line to the material. If material is not included in the article's Creative Commons licence and your intended use is not permitted by statutory regulation or exceeds the permitted use, you will need to obtain permission directly from the copyright holder. To view a copy of this licence, visit http://creativecommons.org/licenses/by/4.0/ The Creative Commons Public Domain Dedication waiver (http://creativecommons.org/publicdomain/zero/1.0/) applies to the data made available in this article, unless otherwise stated in a credit line to the data. 


\section{Background}

The prevalence of obesity is increasing all over the world, in consistence with the increase in obese subjects suffering from infertility [1]. Compared to age-matched normal-weight women, obese women have an increased risk of ovulatory subfertility and anovulatory infertility [2-4]. While ovarian stimulation has been used to overcome anovulation, obese subjects usually require higher doses of gonadotropins. Decreased oocyte retrieval, lower oocyte quality, and reduced rates of preimplantation embryo development are generally observed in obese subjects, in addition to an increased risk of cycle cancellation or miscarriage compared with normal-weight subjects [5-9]. A considerable number of clinical reports have highlighted the negative effect of body mass index (BMI) on the success rate of fertility treatments. However, multiple studies reported no significant association between BMI and assisted reproductive outcomes [10-12]. This controversy indicates that the effect of obesity on oocyte quality and fertility outcome is somewhat complex, and underlying mechanisms remain to be elucidated [13].

Folliculogenesis requires a carefully orchestrated crosstalk between the oocyte and surrounding somatic cells [14]. Two granulosa cell types can be distinguished in the follicle, namely mural granulosa cells (GCs), lying on the basal membrane of the follicular wall, and cumulus GCs, which surround the oocyte [15]. These different GCs provide various molecules, such as sugars, amino acids, signaling factors, and nucleotides, to facilitate oocyte development [16], maturation, meiosis, and oocyte cytoskeleton alteration [17]. Hence, a better understanding of obesity-associated changes in GCs could allow us to identify the mechanisms underlying obesity-induced female infertility.

In the research field of obesity-related female infertility, omics studies were mostly limited to the analysis of follicular fluid or plasma in polycystic ovary syndrome (PCOS) $[18,19]$. However, PCOS is a complex multigenic disorder, which is strongly influenced by various epigenetic and environmental factors [20]. It is therefore difficult to determine the effect of obesity on intrafollicular cell function. The aim of the current study was to investigate how protein expression changes in the GCs of obese women in which PCOS was ruled out as a possible confounder. The results strongly indicate that the GC proteomic expression patterns undergo significant alteration in the obese subjects.

\section{Methods}

\section{Subject enrollment}

This study enrolled subjects visiting the Reproductive Medical Center of Shanghai Ruijin Hospital in vitro fertilization (IVF) Clinics between September 2019 and
January 2020. The study was approved by the local ethics committee of Shanghai Jiao Tong University School of Medicine. All subjects gave informed consent before participation. Twenty-six women with normal menstruation cycles were recruited. The Chinese-specific cutoffs for general adiposity were used, with normal weight as BMI $18.5-23.9 \mathrm{~kg} / \mathrm{m}^{2}$ and general obesity as BMI values equal to or more than $28.0 \mathrm{~kg} / \mathrm{m}^{2}$ [21]. Therefore, subjects with a normal BMI $\left(20.38-21.02 \mathrm{~kg} / \mathrm{m}^{2}\right)(n=12)$ were assigned to the control group, and obese subjects with a BMI of $29.25-32.33 \mathrm{~kg} / \mathrm{m}^{2}(n=14)$ were assigned to the obesity group. The ages of the control group and the obesity group were $31.00 \pm 1.86$ years and $31.79 \pm$ 1.37 years respectively. All participants were Chinese and experienced infertility due to tubal pathology. Subjects with endometriosis, PCOS, or other medical disorders that could affect folliculogenesis were excluded.

\section{Purification of GCs from follicular fluid}

All subjects underwent controlled ovarian stimulation for IVF procedures using standard gonadotropinreleasing hormone antagonist protocols. After receiving human chorionic gonadotrophin injections for 34-36 h, follicular fluid was obtained from follicles $(16-20 \mathrm{~cm}$ in diameter) that contained granulosa cells (GCs). The purification procedure details are as follow: when the mature follicles were obtainable, the cumulus-oocyte complexes were separated from the follicles for IVF treatment, and the remaining follicular fluid was collected and centrifuged at $250 \times \mathrm{g}$ for $10 \mathrm{~min}$. Then the resulting pellet was resuspended with $1 \mathrm{X}$ PBS and subsequently layered on $40 \%$ Percoll $^{\circ}$ (Sigma-Aldrich; Merck $\mathrm{KGaA}$ ) followed by centrifugation at $450 \times \mathrm{g}$ for $20 \mathrm{~min}$ at room temperature. After performing this density gradient centrifugation, the mixture was divided into four layers from the button to the top surface for separating red blood cells, Percoll, granulosa cells and follicular fluid with PBS. The granulosa cell layer was collected and washed with $1 \times \mathrm{PBS}$ at $250 \times \mathrm{g}$ for $10 \mathrm{~min}$. The pellet was resuspended with $1 \mathrm{X}$ PBS, and then the red blood cell lysate was added to a volumetric ratio of $1: 3$ and kept at $4^{\circ} \mathrm{C}$ for $5 \mathrm{~min}$. Finally, three wash steps were carried out using $1 \mathrm{X}$ PBS at $250 \times \mathrm{g}$ for $1 \mathrm{~min}$ at room temperature, and purified GCs were collected.

\section{Total protein extraction}

In order to establish protein content equivalence of $120 \mu \mathrm{g}$ in each sample, the GCs of a single subject whose protein content was less than $120 \mu \mathrm{g}$ were pooled with other GCs belonging to one or two subjects in the same group. This procedure generated 5 control and 5 obese samples in each group. Samples were ground individually in liquid nitrogen and lysed with lysis buffer, followed by ultrasonication on ice for $5 \mathrm{~min}$. The mixture was then 
centrifuged at $12,000 \times \mathrm{g}$ for $15 \mathrm{~min}$ at $4{ }^{\circ} \mathrm{C}$, and the supernatant was collected. Extracts were reduced with 10 mM DTT for $1 \mathrm{~h}$ at $56{ }^{\circ} \mathrm{C}$ individually and were alkylated with iodoacetamide in the absence of light for $1 \mathrm{~h}$ at room temperature. The samples were then mixed with precooled acetone, followed by incubation and centrifugation. Next, the precipitate was collected, washed with cold acetone, and then the pellet was dissolved in dissolution buffer containing $0.1 \mathrm{M}$ triethylammonium bicarbonate (TEAB, $\mathrm{pH} 8.5$ ) and $6 \mathrm{M}$ urea. Finally, the Bradford Protein Assay Kit (Beyotime Biotechnology, China) was used to determine the protein concentrations.

\section{Protein digestion and tandem mass tag (TMT) labeling}

A total of $120 \mu \mathrm{g}$ per protein sample was added to 100 $\mu \mathrm{L}$ of dissolution buffer. Thereafter, $1.5 \mu \mathrm{g}$ trypsin and $500 \mu \mathrm{L}$ of $100 \mathrm{mM}$ TEAB buffer were added, followed by digestion at $37{ }^{\circ} \mathrm{C}$ for $4 \mathrm{~h}$. Subsequently, $1.5 \mu \mathrm{g}$ trypsin and $\mathrm{CaCl}_{2}$ were added, and the sample was digested overnight. Formic acid was mixed with the digested sample, and the $\mathrm{pH}$ was adjusted to $<3$, and then the sample was centrifuged at 12,000 $\times g$ for $5 \mathrm{~min}$. After the supernatant flowing through a $\mathrm{C} 18$ desalting column, the column was washed thrice $(0.1 \%$ formic acid, $4 \%$ acetonitrile) and then eluted with elution buffer ( $0.1 \%$ formic acid, $75 \%$ acetonitrile). The eluents were collected and lyophilized. Next, $100 \mu \mathrm{L}$ of $0.1 \mathrm{M}$ TEAB buffer was added for reconstitution, followed by the addition of $41 \mu \mathrm{L}$ of acetonitrile-dissolved TMT labeling reagent (ThermoFisher, Massachusetts, USA). After mixing the samples by shaking for $2 \mathrm{~h}$, the reaction was stopped by the addition of $8 \%$ ammonia. Then, labeling samples were desalted and lyophilized.

\section{Separation of fractions}

To develop a gradient elution, mobile phase A (2\% acetonitrile, adjusted $\mathrm{pH}$ to 10.0$)$ and $\mathrm{B}$ (98\% acetonitrile) were used. The lyophilized powder was dissolved in solution A. The sample was fractionated using a C18 column, and the column oven was set at $50{ }^{\circ} \mathrm{C}$. At 214 $\mathrm{nm}$, the eluates were monitored, followed by collection into a tube every minute, and eventually combination into 10 fractions. Under vacuum, all fractions were dried and then reconstituted in $0.1 \%(\mathrm{v} / \mathrm{v})$ formic acid in water.

\section{Liquid chromatography tandem mass spectrometry (MS) analysis}

For transition library construction, shotgun proteomics analyses were conducted using an EASY-nLCTM 1200 UHPLC system (Thermo Fisher, Massachusetts, USA) coupled with a $\mathrm{Q}$ Exactive $\mathrm{HF}(\mathrm{X})$ mass spectrometer (Thermo Fisher, Massachusetts, USA). The Q Exactive $\mathrm{HF}(\mathrm{X})$ MS allowed operation in a data-dependent acquisition (DDA) mode.

\section{Identification and quantification of differentially abundant proteins}

The resulting spectra from each run were searched against the SwissProt human database using the Proteome Discoverer v2.2 (ThermoFisher, Massachusetts, USA) search engine. A maximum of two miscleavage sites were allowed. Peptide spectrum matches (PSMs) with a credibility of more than $99 \%$ were identified as PSMs. The identified proteins contained at least one unique peptide. PSMs and proteins with a false discovery rate (FDR) of no more than $1.0 \%$ were retained. Differentially expressed proteins (DEPs) were identified using the limma package [22] with the following criteria: (1) fold change value $>1.2$ or $<1 / 1.2$, (2) $P$ value $<0.05$. DEP analysis was carried out in $\mathrm{R}$ 4.1.0.

\section{Bioinformatics}

Gene Ontology (GO) annotation of identified proteins was performed using the UniProt-GOA database and InterProScan program version 5 [23]. Pathway annotation was carried out using the Kyoto Encyclopedia of Genes and Genomes (KEGG) database. Enrichment analysis of functional annotations was conducted using the R package clusterProfiler [24]. A $P$ value $<0.05$ was considered significant, and enriched GO terms or pathways were sorted by $P$ values. Principle component analysis (PCA) and $\mathrm{t}$-distributed stochastic neighbor embedding ( $\mathrm{t}-\mathrm{SNE}$ ) clustering analyses [25] were conducted using the princomp() function or Rt-SNE package, respectively, in R. Hierarchical clustering analysis of DEPs was performed using the R gplots package. Protein-protein interactions (PPI) were derived using the STRING database [26] (https://string-db.org/). Network visualization of PPI was carried out using Cytoscape version 3.8.0 [27], and hub scores for each identified DEP were obtained using the cytohubba plugin [28]. The modules of hub genes were filtered using the MCODE plugin in Cytoscape with the following criteria: degree cut-off $>2$, node score cut-off $>0.2$, K-score $>2$, and max depth $=10$.

\section{Determination of lipids in serum and follicular fluid}

Venous blood or follicular fluid samples were centrifuged at 3,000 $\times g$ for $10 \mathrm{~min}$ to isolate the upper layer for analysis. Serum sex hormones, including follicle-stimulating hormone, luteinizing hormone, estradiol, progesterone and testosterone, were analyzed using commercially available kits from the Unicel DXI 800 Access immunoassay system (Beckman Coulter). Total cholesterol, triglycerides, high-density lipoprotein, and low-density lipoprotein levels were determined using commercially available kits purchased from Randox Laboratories Ltd., Northern Ireland, United Kingdom. The assays were carried out on a Hitachi 7600-210 Automatic Biochemical Analyzer (Hitachi Corporation, Japan). Commercially available kits 
for free fatty acid (FFA) determination were purchased from Nanjing Jiancheng Bioengineering Institute (Jiangsu, China).

\section{Statistical analysis}

All analyses were performed using $\mathrm{R}$ version 4.1.0. Normally distributed continuous data are presented as the mean \pm SE and were compared using the Student's $t$-test, while non-parametric data were presented as the median (interquartile range) and were compared using the MannWhitney U test, when appropriate. Correlations were evaluated using Spearman's correlation coefficient. A twosided $P$-value $<0.05$ was considered statistically significant for all tests.

\section{Results}

\section{Subject clinical characteristics}

Descriptive statistics and ovarian stimulation characteristics were recorded (Table 1). There was no statistical difference in age, basal hormone levels, or ovarian stimulation procedure data between the two groups. The BMI in the obesity group ranged from 29.25 to $32.33 \mathrm{~kg} / \mathrm{m}^{2}$, much higher than that in the control group (20.38$21.02 \mathrm{~kg} / \mathrm{m}^{2}$ ).

\section{Subject lipid parameters}

Table 2 summarizes lipid levels in the serum and follicular fluid of all participants. FFA and triglycerides levels in follicular fluid and FFA levels in serum of obese women were significantly higher than those in matched normal-weight women. No differences were observed in total cholesterol, high-density lipoprotein, and lowdensity lipoprotein levels between the two groups.

\section{Proteomics analysis}

To obtain a global view of the GC proteome in normalweight and obese subjects, sample clustering based on all identified proteins was performed. As shown in Fig. 1 a, PCA analysis revealed two significant clusters, which indicated distinct protein expression levels in obese subjects. The above result was further confirmed using t-SNE clustering analysis, which also showed two isolated clusters (Fig. 1b). Subsequently, DEPs between the two groups were screened. A total 228 DEPs were identified, including 138 upregulated and 90 downregulated proteins (Fig. 1 c). As shown in Fig. 1 c, the top 10 DEPs were highlighted and labeled, including RNAS EH2C, INSL3, TAPBPL, TSPAN8, TUBB8, and SUMO3. Next, hierarchical clustering based on DEPs was performed, and the results revealed clusters similar to those of PCA and t-SNE analysis (Fig. 1d). The annotation and quantification information for the top 10 DEPs are presented in Table 3.

\section{Changes in the mitochondrial electron transport chain in obese subject GCs}

Functional enrichment analyses, including GO and KEGG, were carried out to investigate the roles of DEPs in GCs and to explore the effect of obesity on oocyte quality and fertility outcome. DEPs were enriched in biological processes related to the mitochondrial respiratory chain, including electron transport, ATP synthesis, cellular respiration, energy derivation by oxidation of organic compounds, and ATP metabolic process (Fig. 2 a). In the cellular component analysis, DEPs were found to be enriched in respirasome, mitochondrial respirasome, endoplasmic reticulum lumen, and other energy metabolism-related

Table 1 Clinical parameters and ovarian stimulation in control and obese groups

\begin{tabular}{|c|c|c|c|}
\hline & Control group $(n=12)$ & Obesity group ( $n=14)$ & $P$-value \\
\hline \multicolumn{4}{|l|}{ Patients information } \\
\hline Age (y) & $31.00 \pm 1.86$ & $31.79 \pm 1.37$ & 0.227 \\
\hline BMI (kg/m2) & $20.75(20.38-21.02)$ & $30.70(29.25-32.33)$ & $<0.001$ \\
\hline \multicolumn{4}{|l|}{ Basal sex hormones (serum) } \\
\hline $\mathrm{FSH}(\mathrm{IU} / \mathrm{L})$ & $8.36 \pm 1.16$ & $8.52 \pm 0.77$ & 0.687 \\
\hline LH(IU/L) & $3.87 \pm 1.21$ & $4.25 \pm 1.14$ & 0.423 \\
\hline $\mathrm{E} 2(\mathrm{pg} / \mathrm{ml})$ & $40.50 \pm 9.06$ & $34.93 \pm 8.40$ & 0.117 \\
\hline $\mathrm{P}(\mathrm{ng} / \mathrm{ml})$ & $0.68 \pm 0.25$ & $0.74 \pm 0.22$ & 0.549 \\
\hline $\mathrm{TT}$ (ng/ml) & $0.37 \pm 0.05$ & $0.40 \pm 0.07$ & 0.207 \\
\hline \multicolumn{4}{|l|}{ Ovarian stimulation cycles } \\
\hline Total FSH dose (IU) & $2806.25 \pm 368.52$ & $2850.00 \pm 314.09$ & 0.747 \\
\hline Duration of stimulation (day) & $10.00(9.00-10.25)$ & 10.00(9.00-10.75) & 0.874 \\
\hline E2 on trigger day $(\mathrm{pg} / \mathrm{ml})$ & $4096.00 \pm 1022.08$ & $3987.29 \pm 1065.23$ & 0.794 \\
\hline LH on trigger day (IU/L) & $3.09 \pm 1.11$ & $2.81 \pm 0.95$ & 0.486 \\
\hline $\mathrm{P}$ on trigger day $(\mathrm{ng} / \mathrm{ml})$ & $1.08 \pm 0.26$ & $1.02 \pm 0.23$ & 0.591 \\
\hline
\end{tabular}


Table 2 Lipids Levels of serum and follicular fluid in control and obese groups

\begin{tabular}{|c|c|c|c|}
\hline & $\begin{array}{l}\text { Control group } \\
(n=12)\end{array}$ & $\begin{array}{l}\text { Obesity group } \\
(n=14)\end{array}$ & $P$-value \\
\hline \multicolumn{4}{|l|}{ Serum } \\
\hline TC (mmol/L) & $4.12 \pm 0.75$ & $4.22 \pm 0.95$ & 0.779 \\
\hline $\mathrm{TG}(\mathrm{mmol} / \mathrm{L})$ & $1.03 \pm 0.34$ & $1.28 \pm 0.41$ & 0.098 \\
\hline $\mathrm{LDL}(\mathrm{mmol} / \mathrm{L})$ & $2.48 \pm 0.48$ & $2.41 \pm 0.49$ & 0.715 \\
\hline $\mathrm{HDL}(\mathrm{mmol} / \mathrm{L})$ & $1.30 \pm 0.10$ & $1.29 \pm 0.12$ & 0.899 \\
\hline FFA (mmol/L) & $0.37 \pm 0.12$ & $0.47 \pm 0.12$ & 0.042 \\
\hline \multicolumn{4}{|l|}{ Follicular fluid } \\
\hline TC (mmol/L) & $0.72 \pm 0.22$ & $0.78 \pm 0.19$ & 0.487 \\
\hline $\mathrm{TG}(\mathrm{mmol} / \mathrm{L})$ & $0.24(0.23-0.26)$ & $0.29(0.26-0.32)$ & 0.01 \\
\hline $\mathrm{FFA}(\mathrm{mmol} / \mathrm{L})$ & $0.32(0.31-0.34)$ & $0.44(0.42-0.46)$ & $<0.001$ \\
\hline
\end{tabular}

subcellular locations (Fig. 2b). Similar results were observed in molecular function analysis, in which electron transfer activity, protease binding, and oxidoreductase activity, among other terms, were significantly enriched (Fig. 2 c).

KEGG pathway analysis revealed that the most significantly enriched pathways included energy metabolism (oxidative phosphorylation, thermogenesis, and carbon metabolism), extracellular matrix (ECM) (ECM-receptor interaction, focal adhesion, and regulation of actin cytoskeleton), steroid hormone synthesis (steroid biosynthesis and ovarian steroidogenesis), diabetes complications (AGE-RAGE signaling pathway in diabetic complications), apoptosis, the PI3K-AKT signaling pathway, and the relaxin (INSL3) signaling pathway, among other terms (Fig. 2d).

\section{Key genes and modules based on the PPI network}

We built the PPI network of DEPs using the STRING database. All DEPs were filtered into the DEP PPI network complex, which contained 189 nodes and 623 edges (Fig. 3 a). Among the 189 nodes, 11 central node genes were selected as hub genes with the criterium of filtering degree $\geq 20$ (i.e., each node having more than 20 connections/interactions). The hub genes were FN1, MAPK3, CYCS, CYC1, NDUFAB1, SDHC, COL1A1, COX4I1, FGF2, COL3A1, and UQCR10. To identify the core gene modules that might play an important role in related pathways, core gene modules were also analyzed, and a total of three hub modules are shown in Fig. 3b-d. Functional enrichment analysis revealed that the genes in hub modules 1, 2, and 3 were mainly associated with "ATP synthesis," "leukocyte migration", and "extracellular matrix organization", respectively (Fig. 3 a).

\section{Discussion}

Obesity and infertility are inextricably linked, and their relationship has been increasingly recognized [29]. As a key factor, GCs play an important role in oocyte and follicle development. They secrete nutrients and hormones essential for primary and secondary follicle growth, providing a suitable microenvironment for oocyte meiosis and maturation [30, 31]. Therefore, a precise understanding of the effect of obesity on GCs may help to determine the mechanism of obesity-related female infertility or subfertility.

The exposure of ovarian cells to high levels of fatty acids could result in an inflammatory response within ovarian follicles [32], excessive ovarian androgen production through ineffective FFA $\beta$-oxidation in mitochondria [33], insulin resistance [34], and oxidative DNA damage caused by reactive oxygen species (ROS) reacting with DNA within the cell, including mitochondrial DNA (mtDNA) [35]. In the current study, the concentration of FFA and triglycerides in the follicular fluid of obese subjects was higher than that in control group. This was consistent with the results of our previous study, in which we found that FFA and triglycerides, as important biomedical indicators of abnormal lipid metabolism, were elevated in the serum and follicular fluid of obese subjects with PCOS and associated with lower embryo quality [36].

Mass spectrometry is widely used in proteomics for accurate and high-throughput analysis, allowing for the identification and quantification of proteins in complex samples with high sensitivity [37]. In this study, we used TMT-based high-throughput MS to investigate the proteome of GC samples from normal-weight and obese subjects. DEPs between the two groups were screened, and a total of 228 DEPs were identified, including 138 upregulated and 90 downregulated proteins. Significant pathways and GO terms associated with protein expression changes were also identified, especially those associated with the mitochondrial electron transport chain. With regard to the biological process category, almost all of the significant GO terms were associated with the mitochondrial respiratory chain. 

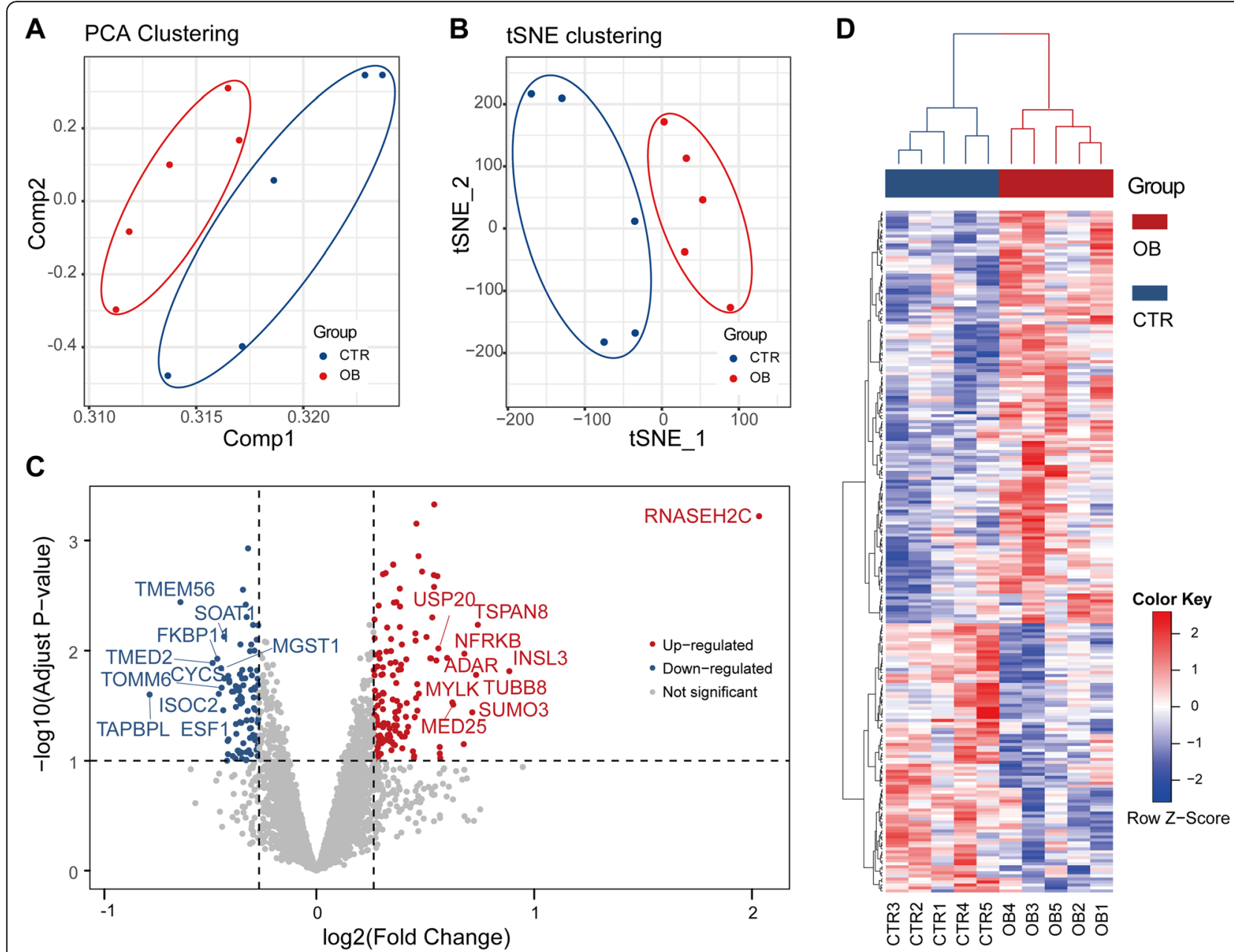

Fig. 1 Quantitative analysis of proteomics data between control group and obese subjects. a Scatter plot showing the principle component analysis clustering of subjects. Each dot represents a subject and is colored according to subject groups indicated in the bottom-right corner of the plot. b Scatter plot showing the t-distributed stochastic neighbor embedding clustering of subjects. c Volcano plot of differentially expressed proteins (DEPs). The $x$-axis corresponds to the log2-transformed fold change of DEPs identified when comparing obese group (OB) versus control group (CTR). The y-axis corresponds to -log10-transformed $P$ values. Upregulated and downregulated DEPs are colored in red and blue, respectively. The top 10 upregulated and downregulated DEPs are labeled. $\mathbf{d}$ Heatmap showing the hierarchical clustering of samples and DEPs. Samples of the OB and CTR groups are colored in red and blue, respectively

Caloric excess and high-fat diet is the most common cause of obesity. High-fat diet can lead to nutritional excess, increased electron flux, rising oxidative stress, an accumulation of oxidized substrates, and eventually, mitochondrial injury [38]. Physiologically, the mitochondrial matrix flowing within the inner membrane, which hosts several copies mtDNA as well as components of the tricarboxylic acid pathway and electron transport chain, is the site of ATP production [39]. Excess FFA can increase mitochondrial activity, contributing to higher oxidative stress, which adversely affects the replication of mtDNA and leads to the dysfunction or dysregulation of key mtDNA gene products. Subsequently, mtDNA gene products control oxidative phosphorylation, thereby impairing the maturation and developmental potential of oocytes [40]. Damage to mitochondrial function caused by high concentrations of FFA has been confirmed by both in vivo and in vitro studies in multiple tissue and cell types [41-43]. In the current study, a significant correlation between high FFA in follicular fluid and mitochondrial dysfunction in GCs from obese subjects was observed. Among molecular function GO terms, electron transfer activity, which is essential for mitochondrial energy production, was listed as the most significantly perturbed function. In addition, cytochrome-c-oxidase activity, which is also key factor for the respiratory electron transport chain, was altered in GCs from obese subjects.

The ECM is a diverse mixture of molecules, influencing almost all aspects of the follicle development 
Table 3 List of differentially expressed proteins identified in GCs obtained from obese women and control group

\begin{tabular}{lllll}
\hline Protein Accession & Gene & Description & Fold change & P-Value \\
\hline E9PN81 & RNASEH2C & Ribonuclease H2 subunit C & 4.09 & 1.85 \\
P51460 & INSL3 & Insulin-like 3 & 0.0001 \\
Q9BX59 & TAPBPL & Tapasin-related protein & 1.67 & 0.02 \\
P19075 & TSPAN8 & Tetraspanin-8 & 1.66 & 0.03 \\
Q3ZCM7 & TUBB8 & Tubulin beta-8 chain & 1.64 & 0.01 \\
A8MU27 & SUMO3 & Small ubiquitin-related modifier 3 & 1.6 & 0.02 \\
A0A024R3N2 & NFRKB & Nuclear factor related to kappaB binding protein, isoform CRA_a & 1.6 & 0.04 \\
Q96GX5 & MASTL & Serine/threonine-protein kinase greatwall & 1.55 & 0.01 \\
Q71SY5 & MED25 & Mediator of RNA polymerase II transcription subunit 25 & 1.54 & 0.03 \\
Q15746 & MYLK & Myosin light chain kinase, smooth muscle & 0.03
\end{tabular}

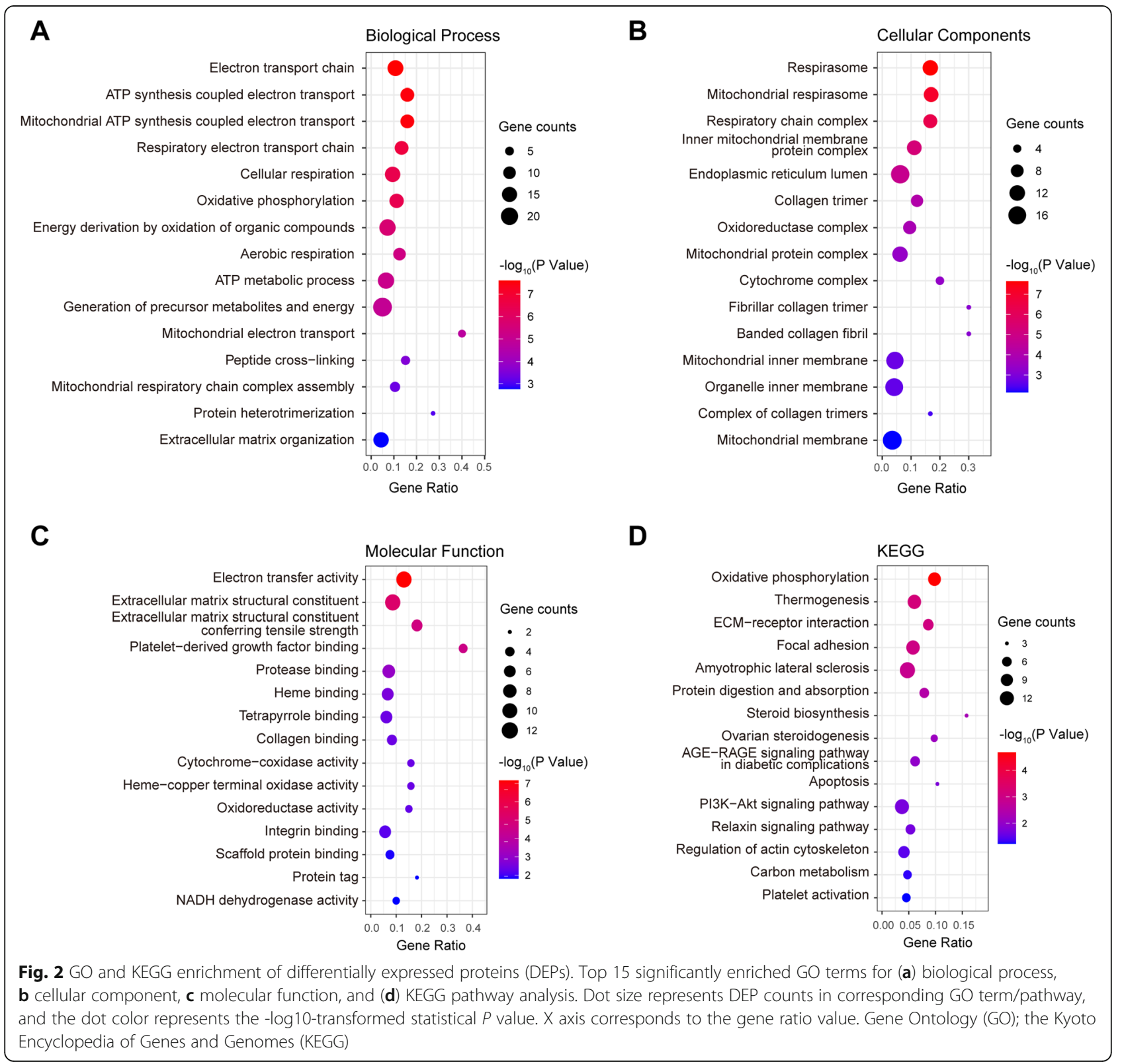




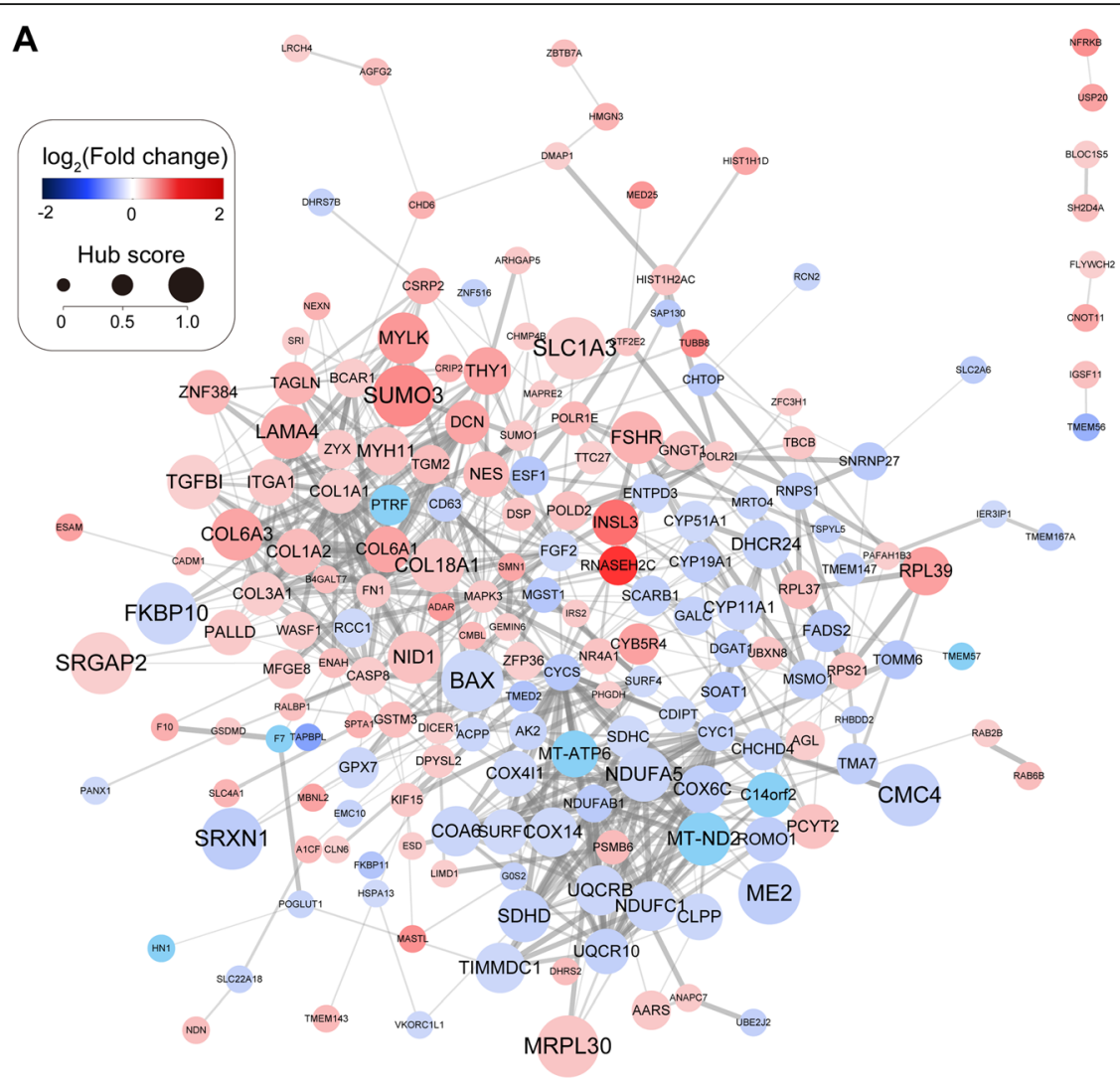

B

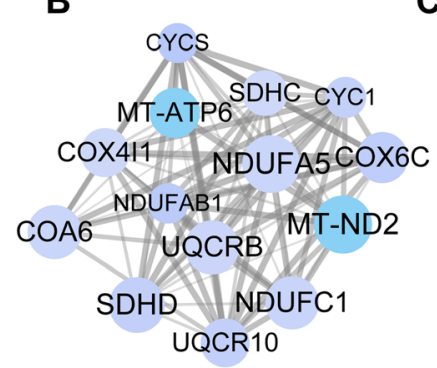

C

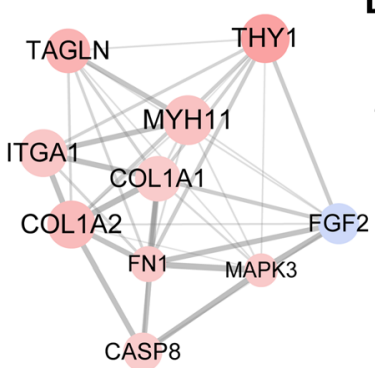

D

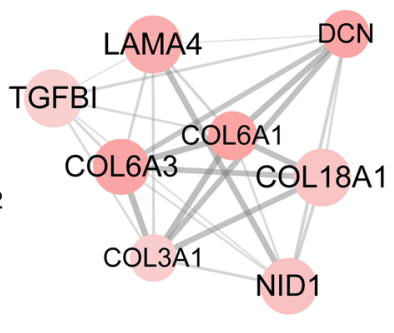

Fig. 3 Protein-protein interactions (PPI) network of differentially expressed proteins (DEPs). a PPI network of all DEPs. DEPs are illustrated as dots, and edges between DEPs indicate potential interactions. Dot color corresponds to the log2-transformed fold change (obese group versus control group), while dot size and dot label size correspond to the clustering coefficient. Edge width corresponds to the combined interaction score predicted by the STRING database. $\mathbf{b}, \mathbf{c}$, $\mathbf{d}$ represent the three core modules identified by Cytoscape

process, including migration, division, differentiation, cell death, and cell anchorage [44]. Various ECM types in the ovarian follicles have been characterized, including those associated with the follicular basal lamina, the stromal matrix of the thecal layers, the matrix of thecal blood vessels, and that of the cumulus oocyte complexes [45-47]. Changes relevant to the structure of the ECM were related to a large part of the obtained molecular function GO terms, including changes in extracellular matrix structural constituent, extracellular matrix structural constituent conferring tensile strength, protease binding, collagen binding, and scaffold protein binding. The current study findings indicated that obesity and high FFA levels may affect the function of ovarian GCs via alteration of the ECM.

The regulation of endoplasmic reticulum homeostasis is indispensable during folliculogenesis and oocyte maturation [48]. Some physiological and pathological conditions, such as glucose deprivation, inflammation, oxidative stress, elevated FFA levels, aberrant $\mathrm{Ca}^{2+}$ regulation, and hypoxia disrupt endoplasmic reticulum function, which results in the accumulation of unfolded or 
misfolded proteins within the endoplasmic reticulum, leading to the induction of endoplasmic reticulum stress [49]. Fatty acid-induced endoplasmic reticulum stress in mouse cumulus-oocyte complexes impairs protein secretion and mitochondrial activity, resulting in abnormal embryo development [50]. Another mouse study also demonstrated a relationship between obesity and endoplasmic reticulum stress in cumulus-oocyte complexes, which was associated with reduced mitochondrial membrane potential, high autophagy levels, and high intracellular lipid levels [51]. The mechanisms underlying endoplasmic reticulum changes in the GCs of obese female subjects in this study may be similar to those reported in animal studies $[50,51]$.

Regarding the cellular component category, the major enriched terms were related to the mitochondria, ECM, and endoplasmic reticulum, which were also consistent with the results for the biological process and molecular function categories. The mitochondria-related terms included mitochondrial respirasome, respiratory chain complex, cytochrome complex, and oxidoreductase complex. ECM terms included collagen trimer, fibrillar collagen trimer, banded collagen fibril, and complex of collagen trimers. Endoplasmic reticulum terms included endoplasmic reticulum lumen.

Several studies have shown that oxidative stress caused by increased levels of ROS can induce GC apoptosis, resulting in decreased estradiol $17 \beta$ levels, lower ovulation rates, and compromised oocyte quality [52]. In the KEGG pathway analysis, energy metabolism and ECMrelated pathways were among the most significantly enriched, which were consistent with GO results. Thus, oxidative stress caused by mitochondrial dysfunction and a high fatty acid environment may affect steroid synthesis, in turn affecting the quality of oocytes.

In patients with PCOS, advanced glycation endproduct (AGE) levels were significantly increased compared with those in matched normal-weight controls $[53,54]$. AGEs can alter insulin receptor substrate and AKT phosphorylation, thereby stimulating the downstream PI3K signaling cascade and compromising the effects of insulin signaling $[55,56]$. Excessive deposition of AGEs can impair the ECM structure, altering its biochemical characteristics and associated metabolism [57]. The binding of AGE to its receptor RAGE produces $\mathrm{NAPDH}$, which enhances oxidative stress, activates the NF- $\mathrm{KB}$ signaling pathway and further stimulates the production of cytokines and other factors. It finally contributes to cellular damage and even apoptosis [58, 59]. Moreover, the PI3K-AKT signaling pathway was associated with human GC damage and apoptosis [60, 61]. An interesting result of the KEGG pathway analysis was the significant enrichment of the AGE-RAGE signaling pathway in the current study. This is the first to demonstrate the association between AGEs and impaired GC function in obese non-PCOS subjects. It suggests that obesityrelated infertility or subfertility is closely associated with the metabolic, inflammatory, structural, and functional changes in GCs.

TSPAN8 is a cell surface glycoprotein, which forms complexes with integrins. Previous genome-wide association studies identified TSPAN8 loci, which was correlated with an increased risk of type 2 diabetes (T2D) in humans [62-64]. Close examination of the haploblock structure with the inclusion of additional SNPs suggested that TSPAN8 is likely to be a T2D causal gene [65]. Genetic ablation of TSPAN8 resulted in a reduction of body weight in mice fed a normal diet as well as in a resistance to body weight gain upon highfat and high-carbohydrate diet feeding [65]. Small ubiquitin-like modifier 3 (SUMO3) is a member of the SUMO family of eukaryotic proteins. Subcutaneous adipose tissue from cows incubated with insulin exhibited an upregulation of SUMO3, in parallel to an increased expression of other key genes associated with insulin signaling, adipogenesis, and lipogenesis [66]. In the field of reproduction, previous literature reported that excessive SUMOylation (SUMO1/2/3) was a marker of defective spermatozoa [67]. INSL3 is a member of the insulin-like hormone superfamily and is mainly produced in gonadal tissues. Within the follicle, INSL3 acts via its G proteincoupled receptor, RXFP2, in an autocrine/paracrine manner to drive the production of the major steroid precursor androstenedione as well as its conversion into estrogen by GCs [68]. MED25 was previously shown to play a critical role in the endoplasmic reticulum stress response [69] Here, a number of the DEPs identified in the current work, including TSPAN8, SUMO3, INSL3, and MED25, were implicated in obesity-related infertility or subfertility. In addition, INSL3 was one of the most differentially expressed proteins, which was consistent with the enrichment of the relaxin signaling pathway in KEGG analysis. Other major DEPs, including RNAS EH2C, TAPBPL, TUBB8, NFRKB, MASTL, and MYLK, have never been reported to be associated with infertility, metabolic disorders, or hormone imbalances. Thus, their role in the occurrence and development of female infertility requires further attention.

\section{Conclusions}

In summary, TMT-based proteomic and bioinformatic analyses indicated that mitochondrial damage, the endoplasmic reticulum stress response as well as dysregulated hormonal synthesis were exhibited in GCs obtained from obese subjects whereas none of these changes occurred in normal-weight subjects. These alterations may be related to the high FFA and TG levels detected in human follicular fluid. Therefore, the current research 
provides valuable insight for the further etiological investigation of obesity-related infertility or subfertility and the identification of therapeutic targets.

\section{Abbreviations}

BMI: Body mass index; TC: Total cholesterol; TG: Triglycerides; HDL: Highdensity lipoprotein; LDL: Low-density lipoprotein; FFA: Free fatty acid; PCA: Principle component analysis; t-SNE: t-distributed stochastic neighbor embedding; PPI: Protein-protein interactions; PSMs: Peptide spectrum matches; GCs: Granulosa cells; CTR: Control group; OB: Obesity group; IVF: In vitro fertilization; TEAB: Triethylammonium bicarbonate; DEP: Differentially expressed protein; GO: Gene Ontology; KEGG: Kyoto Encyclopedia of Genes and Genomes; SY: Symmetry; PCOS: Polycystic ovary syndrome; mtDNA: Mitochondrial DNA; ROS: Reactive oxygen species; ECM: Extracellular matrix; ER: Endoplasmic reticulum; SUMO: Small ubiquitinrelated modifier; AGE: Advanced glycation end-product; MF: Molecular function; CC: Cellular component; BP: Biological process; MS: Mass spectrometry; TMT: Tandem mass tag; OS: Oxidative stress

\section{Acknowledgements}

The authors thank Prof. Peter Reinach for his editorial assistance.

\section{Authors' contributions}

NZ and SC designed the study. SC and WN performed the experiments. WM contributed significantly to the data analysis. LY and DZ helped with constructive discussions. SC wrote the initial manuscript. NZ and DZ supervised and supported the whole project, and edited the manuscript. DZ provided final approval of the manuscript. All authors read and agreed on the final version of the manuscript.

\section{Funding}

This research project was financially supported by grants from the National Natural Science Foundation of China (No. 82071694, 81671530) and Science and Technology Commission of Shanghai Municipality (No. 21140904000, 201409005800).

\section{Availability of data and materials}

The datasets used and analyzed during the current study are available from the corresponding author on reasonable request.

\section{Declarations}

\section{Ethics approval and consent to participate}

All participants signed informed consent forms approved by the Shanghai Jiao Tong University Committee on the Use of Human Subjects in Medical Research. Ethical approval of follicular fluid collection was obtained from the institutional Ethics Committee of Ruijin Hospital, School of Medicine, Shanghai Jiao Tong University.

\section{Consent for publication}

Not applicable.

\section{Competing interests}

The authors declare that they have no competing interests.

Received: 18 February 2021 Accepted: 10 May 2021

Published online: 20 May 2021

\section{References}

1. Best D, Avenell A, Bhattacharya S. How effective are weight-loss interventions for improving fertility in women and men who are overweight or obese? A systematic review and meta-analysis of the evidence. Hum Reprod Update. 2017;23:681-705.

2. Pandey S, Bhattacharya S. Impact of obesity on gynecology. Womens Health (Lond). 2010;6:107-17.

3. Kumbak B, Oral E, Bukulmez O. Female obesity and assisted reproductive technologies. Semin Reprod Med. 2012;30:507-16.

4. Penzias AS. Recurrent IVF failure: other factors. Fertil Steril. 2012;97:1033-8.
5. Broughton DE, Moley KH. Obesity and female infertility: potential mediators of obesity's impact. Fertil Steril. 2017;107:840-47.

6. Shah DK, Missmer SA, Berry KF, Racowsky C, Ginsburg ES. Effect of obesity on oocyte and embryo quality in women undergoing in vitro fertilization. Obstet Gynecol. 2011;118:63-70.

7. Provost MP, Acharya KS, Acharya CR, Yeh JS, Steward RG, Eaton JL, Goldfarb $J M$, Muasher SJ. Pregnancy outcomes decline with increasing body mass index: analysis of 239,127 fresh autologous in vitro fertilization cycles from the 2008-2010 Society for Assisted Reproductive Technology registry. Fertil Steril. 2016;105:663-69.

8. Wang JX, Davies M, Norman RJ. Body mass and probability of pregnancy during assisted reproduction treatment: retrospective study. BMJ. 2000;321: 1320-1.

9. Sermondade N, Huberlant S, Bourhis-Lefebvre V, Arbo E, Gallot V, Colombani M, Fréour T. Female obesity is negatively associated with live birth rate following IVF: a systematic review and meta-analysis. Hum Reprod Update. 2019;25:439-51.

10. Lashen $H$, Ledger W, Bernal AL, Barlow D. Extremes of body mass do not adversely affect the outcome of superovulation and in-vitro fertilization. Human reproduction (Oxford England). 1999;14:712-15.

11. Dechaud H, Anahory T, Reyftmann L, Loup V, Hamamah S, Hedon B. Obesity does not adversely affect results in patients who are undergoing in vitro fertilization and embryo transfer. Eur J Obstet Gynecol Reprod Biol. 2006; 127:88-93.

12. Martinuzzi K, Ryan S, Luna M, Copperman AB. Elevated body mass index (BMI) does not adversely affect in vitro fertilization outcome in young women. J Assist Reprod Genet. 2008:25:169-75.

13. Valckx SDM, De Pauw I, De Neubourg D, Inion I, Berth M, Fransen E, Bols PEJ, Leroy JLMR. BMI-related metabolic composition of the follicular fluid of women undergoing assisted reproductive treatment and the consequences for oocyte and embryo quality. Human reproduction (Oxford, England). 2012; 27:3531-39.

14. Uyar A, Torrealday S, Seli E. Cumulus and granulosa cell markers of oocyte and embryo quality. Fertil Steril. 2013;99:979-97.

15. Chronowska E. High-throughput analysis of ovarian granulosa cell transcriptome. Biomed Res Int. 2014; 2014:213570.

16. Russell DL, Gilchrist RB, Brown HM, Thompson JG. Bidirectional communication between cumulus cells and the oocyte: Old hands and new players? Theriogenology. 2016;86:62-8.

17. Coticchio G, Dal Canto M, Mignini Renzini M, Guglielmo MC, Brambillasca F, Turchi D, Novara PV, Fadini R. Oocyte maturation: gamete-somatic cells interactions, meiotic resumption, cytoskeletal dynamics and cytoplasmic reorganization. Hum Reprod Update. 2015;21:427-54.

18. Sun L, Hu W, Liu Q, Hao Q, Sun B, Zhang Q, Mao S, Qiao J, Yan X. Metabonomics reveals plasma metabolic changes and inflammatory marker in polycystic ovary syndrome patients. J Proteome Res. 2012;11:2937-46.

19. Zhang X, Xu X, Li P, Zhou F, Kong L, Qiu J, Yuan Z, Tan J. TMT Based Proteomic Analysis of Human Follicular Fluid From Overweight/Obese and Normal-Weight Patients With Polycystic Ovary Syndrome. Front Endocrinol. 2019;10:821

20. Escobar-Morreale HF. Polycystic ovary syndrome: definition, aetiology, diagnosis and treatment. Nature reviews Endocrinology. 2018;14:270-84.

21. Consultation WHOE. Appropriate body-mass index for Asian populations and its implications for policy and intervention strategies. Lancet. 2004;363: 157-63.

22. Ritchie ME, Phipson B, Wu D, Hu Y, Law CW, Shi W, Smyth GK. limma powers differential expression analyses for RNA-sequencing and microarray studies. Nucleic Acids Res. 2015;43:e47.

23. Jones $P$, Binns D, Chang HY, Fraser M, Li W, McAnulla C, McWilliam H, Maslen J, Mitchell A, Nuka G, et al. InterProScan 5: genome-scale protein function classification. Bioinformatics. 2014;30:1236-40.

24. Yu G, Wang LG, Han Y, He QY. clusterProfiler: an R package for comparing biological themes among gene clusters. OMICS. 2012;16:284-7.

25. van der Maaten L, Hinton G. Visualizing Data using t-SNE. Journal of Machine Learning Research. 2008;9:2579-605.

26. Szklarczyk D, Gable AL, Lyon D, Junge A, Wyder S, Huerta-Cepas J, Simonovic M, Doncheva NT, Morris JH, Bork P, et al. STRING v11: proteinprotein association networks with increased coverage, supporting functional discovery in genome-wide experimental datasets. Nucleic Acids Res. 2019; 47:D607-D13. 
27. Shannon P, Markiel A, Ozier O, Baliga NS, Wang JT, Ramage D, Amin N, Schwikowski B, Ideker T. Cytoscape: a software environment for integrated models of biomolecular interaction networks. Genome Res. 2003;13:2498-504.

28. Chin $\mathrm{CH}$, Chen $\mathrm{SH}, \mathrm{Wu} \mathrm{HH}, \mathrm{Ho} \mathrm{CW}$, Ko MT, Lin CY. cytoHubba: identifying hub objects and sub-networks from complex interactome. BMC Syst Biol. 2014;8(Suppl 4):11.

29. Talmor A, Dunphy B. Female obesity and infertility. Best Pract Res Clin Obstet Gynaecol. 2015;29:498-506.

30. Komatsu K, Masubuchi S. Mouse oocytes connect with granulosa cells by fusing with cell membranes and form a large complex during follicle development. Biol Reprod. 2018;99:527-35.

31. Ernst EH, Lykke-Hartmann K. Transcripts encoding free radical scavengers in human granulosa cells from primordial and primary ovarian follicles. J Assist Reprod Genet. 2018:35:1787-98.

32. Gonzalez MB, Lane M, Knight EJ, Robker RL. Inflammatory markers in human follicular fluid correlate with lipid levels and Body Mass Index. J Reprod Immunol. 2018:130:25-9.

33. Gervais A, Battista MC, Carranza-Mamane B, Lavoie HB, Baillargeon JP. Follicular fluid concentrations of lipids and their metabolites are associated with intraovarian gonadotropin-stimulated androgen production in women undergoing in vitro fertilization. J Clin Endocrinol Metab. 2015;100:1845-54.

34. Xu L, Wang W, Zhang X, Ke H, Qin Y, You L, Li W, Lu G, Chan WY, Leung PCK, et al. Palmitic acid causes insulin resistance in granulosa cells via activation of JNK. J Mol Endocrinol. 2019;62:197-206.

35. Bradley J, Swann K. Mitochondria and lipid metabolism in mammalian oocytes and early embryos. Int J Dev Biol. 2019;63:93-103.

36. Niu Z, Lin N, Gu R, Sun Y, Feng Y. Associations between insulin resistance, free fatty acids, and oocyte quality in polycystic ovary syndrome during in vitro fertilization. J Clin Endocrinol Metab. 2014;99:E2269-76.

37. Li S, Tang H. Computational Methods in Mass Spectrometry-Based Proteomics. Adv Exp Med Biol. 2016;939:63-89.

38. Bhatraju NK, Agrawal A. Mitochondrial Dysfunction Linking Obesity and Asthma. Ann Am Thorac Soc. 2017;14:368-S73.

39. Kimura I, Ichimura A, Ohue-Kitano R, Igarashi M. Free Fatty Acid Receptors in Health and Disease. Physiol Rev. 2020;100:171-210.

40. Grindler NM, Moley KH. Maternal obesity, infertility and mitochondrial dysfunction: potential mechanisms emerging from mouse model systems. Mol Hum Reprod. 2013:19:486-94.

41. Sacca SC, Cutolo CA, Ferrari D, Corazza P, Traverso CE. The Eye, Oxidative Damage and Polyunsaturated Fatty Acids. Nutrients. 2018; 10.

42. Harasymowicz NS, Dicks A, Wu CL, Guilak F. Physiologic and pathologic effects of dietary free fatty acids on cells of the joint. Ann N Y Acad Sci. 2019;1440:36-53.

43. Reis AH. Acidemia and blood free fatty acids: analysis of cardiovascular risk factors in a new context. Discov Med. 2017;23:183-88.

44. Irving-Rodgers HF, Rodgers RJ. Extracellular matrix in ovarian follicular development and disease. Cell Tissue Res. 2005;322:89-98.

45. Rodgers RJ, Irving Rodgers HF. Extracellular matrix of the bovine ovarian membrana granulosa. Mol Cell Endocrinol. 2002;191:57-64.

46. Rodgers RJ, Irving-Rodgers HF, Russell DL. Extracellular matrix of the developing ovarian follicle. Reproduction. 2003;126:415-24.

47. Rodgers RJ, Irving-Rodgers HF, van Wezel IL. Extracellular matrix in ovarian follicles. Mol Cell Endocrinol. 2000;163:73-9.

48. Guzel E, Arlier S, Guzeloglu-Kayisli O, Tabak MS, Ekiz T, Semerci N, Larsen K, Schatz F, Lockwood CJ, Kayisli UA. Endoplasmic Reticulum Stress and Homeostasis in Reproductive Physiology and Pathology. Int J Mol Sci. 2017; 18

49. Huang N, Yu Y, Qiao J. Dual role for the unfolded protein response in the ovary: adaption and apoptosis. Protein Cell. 2017:8:14-24.

50. Wu LL, Russell DL, Norman RJ, Robker RL. Endoplasmic reticulum (ER) stress in cumulus-oocyte complexes impairs pentraxin-3 secretion, mitochondrial membrane potential (DeltaPsi m), and embryo development. Mol Endocrinol. 2012:26:562-73.

51. Wu LL, Russell DL, Wong SL, Chen M, Tsai TS, St John JC, Norman RJ, Febbraio MA, Carroll J, Robker RL. Mitochondrial dysfunction in oocytes of obese mothers: transmission to offspring and reversal by pharmacological endoplasmic reticulum stress inhibitors. Development. 2015;142:681-91.

52. Chaube SK, Shrivastav TG, Tiwari M, Prasad S, Tripathi A, Pandey AK. Neem (Azadirachta indica L.) leaf extract deteriorates oocyte quality by inducing ROS-mediated apoptosis in mammals. Springerplus. 2014;3:464.
53. Azhary JMK, Harada M, Kunitomi C, Kusamoto A, Takahashi N, Nose E, Oi N, Wada-Hiraike O, Urata Y, Hirata T, et al. Androgens Increase Accumulation of Advanced Glycation End Products in Granulosa Cells by Activating ER Stress in PCOS. Endocrinology. 2020; 161.

54. Diamanti-Kandarakis E, Piperi C, Patsouris E, Korkolopoulou P, Panidis D, Pawelczyk L, Papavassiliou AG, Duleba AJ. Immunohistochemical localization of advanced glycation end-products (AGEs) and their receptor (RAGE) in polycystic and normal ovaries. Histochem Cell Biol. 2007;127:581-9.

55. Wu CH, Huang HW, Huang SM, Lin JA, Yeh CT, Yen GC. AGE-induced interference of glucose uptake and transport as a possible cause of insulin resistance in adipocytes. J Agric Food Chem. 2011:59:7978-84.

56. Diamanti-Kandarakis E, Chatzigeorgiou A, Papageorgiou E, Koundouras D, Koutsilieris M. Advanced glycation end-products and insulin signaling in granulosa cells. Exp Biol Med (Maywood). 2016;241:1438-45.

57. Bansode SB, Gacche RN. Glycation-induced modification of tissue-specific ECM proteins: A pathophysiological mechanism in degenerative diseases. Biochim Biophys Acta Gen Subj. 2019;1863:129411.

58. Wautier MP, Chappey O, Corda S, Stern DM, Schmidt AM, Wautier JL. Activation of NADPH oxidase by AGE links oxidant stress to altered gene expression via RAGE. Am J Physiol Endocrinol Metab. 2001;280:E685-94.

59. Faria A, Persaud SJ. Cardiac oxidative stress in diabetes: Mechanisms and therapeutic potential. Pharmacol Ther. 2017:172:50-62.

60. Zhao Y, Zhang C, Huang Y, Yu Y, Li R, Li M, Liu N, Liu P, Qiao J. Upregulated expression of WNT5a increases inflammation and oxidative stress via PI3K/AKT/NF-kappaB signaling in the granulosa cells of PCOS patients. J Clin Endocrinol Metab. 2015:100:201-11.

61. Nakahara T, Iwase A, Nakamura T, Kondo M, Bayasula, Kobayashi H, Takikawa S, Manabe S, Goto M, Kotani T, Kikkawa F. Sphingosine-1-phosphate inhibits H2O2-induced granulosa cell apoptosis via the PI3KJAkt signaling pathway. Fertil Steril. 2012;98:1001-8 e1.

62. Stuebe AM, Lyon H, Herring AH, Ghosh J, Wise A, North KE, Siega-Riz AM. Obesity and diabetes genetic variants associated with gestational weight gain. Am J Obstet Gynecol. 2010;203:283 e1-17.

63. Kong $X$, Zhang $X$, Xing $X$, Zhang B, Hong J, Yang W. The Association of Type 2 Diabetes Loci Identified in Genome-Wide Association Studies with Metabolic Syndrome and Its Components in a Chinese Population with Type 2 Diabetes. PLoS One. 2015;10:e0143607.

64. Zhou DZ, Liu Y, Zhang D, Liu SM, Yu L, Yang YF, Zhao T, Chen Z, Kan MY, Zhang ZF, et al. Variations in/nearby genes coding for JAZF1, TSPAN8/LGR5 and HHEX-IDE and risk of type 2 diabetes in Han Chinese. J Hum Genet. 2010;55:810-5.

65. Champy MF, Le Voci L, Selloum M, Peterson LB, Cumiskey AM, Blom D. Reduced body weight in male Tspan8-deficient mice. Int J Obes (Lond). 2011:35:605-17.

66. Lopreiato V, Hosseini A, Rosa F, Zhou Z, Alharthi A, Trevisi E, Loor JJ. Dietary energy level affects adipose depot mass but does not impair in vitro subcutaneous adipose tissue response to short-term insulin and tumor necrosis factor-alpha challenge in nonlactating, nonpregnant Holstein cows. J Dairy Sci. 2018;101:10206-19.

67. Vigodner M, Shrivastava V, Gutstein LE, Schneider J, Nieves E, Goldstein M, Feliciano M, Callaway M. Localization and identification of sumoylated proteins in human sperm: excessive sumoylation is a marker of defective spermatozoa. Hum Reprod. 2013;28:210-23.

68. Ivell R, Anand-Ivell R. Insulin-like peptide 3 (INSL3) is a major regulator of female reproductive physiology. Hum Reprod Update. 2018;24:639-51.

69. Sela D, Conkright JJ, Chen L, Gilmore J, Washburn MP, Florens L, Conaway RC, Conaway JW. Role for human mediator subunit MED25 in recruitment of mediator to promoters by endoplasmic reticulum stress-responsive transcription factor ATF6alpha. J Biol Chem. 2013:288:26179-87.

\section{Publisher's Note}

Springer Nature remains neutral with regard to jurisdictional claims in published maps and institutional affiliations. 